\title{
Left Sided Gastroschisis
}

\author{
Sirajuddin Soomro*
}

Department of Paediatric Surgery Chandka Medical College Larkana, Pakistan

\author{
Correspondence*: Sirajuddin Soomro, Department of Paediatric Surgery Chandka Medical College Larkana, Pakistan. \\ E-mail: soomrosiraj@yahoo.com \\ Submitted: 22-07-2017 \\ Conflict of Interest: None \\ (C) 2017, Soomro \\ Accepted: 02-08-2017 \\ Source of Support: Nil
}

This is an open-access article distributed under the terms of the Creative Commons Attribution License, which permits unrestricted use, distribution, and reproduction in any medium, provided the original work is properly cited.

\section{DEAR SIR}

The classical gastroschisis is a defect in abdominal wall on right side of the umbilical cord through which the loops of intestine herniate. The gastroschisis occurring on left side of the umbilical cord is rarely reported.[1]

A 30 -hour old female baby, weighing $1.7 \mathrm{Kg}$, product of consanguineous marriage, born through normal vaginal delivery at home at 36th week of gestation, to a young non-smoker mother presented with evisceration of loops of intestine through a defect in the anterior abdominal wall. On examination the baby was hypothermic, lethargic with bluish color of extremities. On abdominal examination a defect in the anterior abdominal wall, about $2 \mathrm{~cm}$ on left of umbilical cord was noted. Eviscerated exposed loops of intestine were dry, matted with each other, and had multiple black colored patches (Fig.1).

A murmur was audible. Rest of the systemic examination was unremarkable. Patient's general condition was stabilized by providing warm environment in an incubator. A nasogastric tube was passed for aspiration, warm $\mathrm{i} / \mathrm{v}$ fluids started and antibiotics given. After resuscitation the patient was shifted in operation theater for surgery under general anesthesia. The defect was enlarged by applying incisions on superior and inferior aspect of the defect. The umbilical stump excised and contents of umbilical cord examined. There was one opening of umbilical vein and two openings of the umbilical arteries. The matted loops of intestine separated and examined to rule out any area of atresia. The eviscerated loops identified as loops of small intestine (jejunum and ileum) only. The abdominal cavity found under developed not able to house all of the prolapsed intestine even after stretching of the abdominal wall, hence a plastic bag (blood collection bag) applied to create a silo and fixed with anterior abdominal wall. The postoperative course was stormy and patient developed septicemia and expired on 3rd postoperative day.

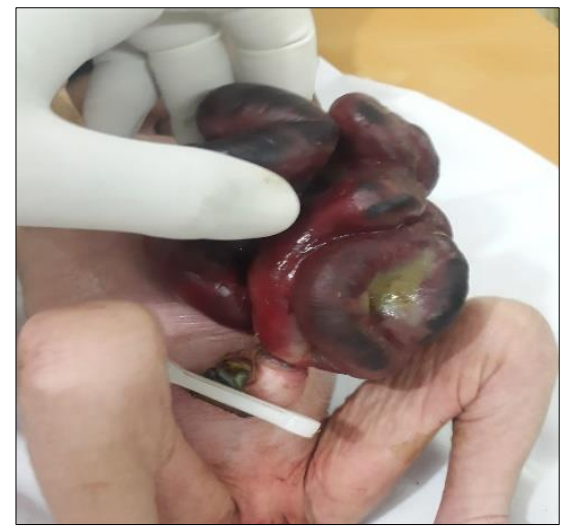

Figure 1: Left sided gastroschisis.

Left sided gastroschisis is an extremely rare event with only 23 cases reported in the literature.[2] Majority of the reported cases of left sided gastroschisis occurred in female babies born to young age mothers.[3-5] Our patient was also a female and born to a young 18 year old primigravida. Over $90 \%$ cases of gastroschisis can now be diagnosed on antenatal ultrasound examination of the 
mother but same was not picked up on antenatal ultrasound examination in our patient.

The left sided gastroschisis is associated with other congenital anomalies more frequently than the right sided lesions. This include cardiac defects, situs inversus, ectopic anus, cecal agenesis, and choledochal cysts. The other associations include macrocephaly, scoliosis and cerebral arterio-venous malformations.[2] Our patient had associated ventricular septal defect. More than $90 \%$ of patients with gastroschisis survive.[5] In our patient sepsis resulted in early demise.

Consent: Author has submitted signed consent form from legal guardians of the patient for use of clinical material in this manuscript. The Consent form is available with Editorial office.

\section{REFERENCES}

1. Orpen NM, Mathievathaniy M, Hitchcock R. Left sided gastroschisis and pseudoexstrophy: a rare combination of anomalies. Pediatr Surg Int. 2004; 20:551-2.

2. Rahul SK, Yadav R, Kumar V, Thakur VK, Hasan Z, Agarwal A. Left sided gastroschisis. J Neonatal Surg. 2017;6:53.

3. Holland AJ, Walker K, Badawi N. Gastroschisis: an update. Pediatr Surg Int. 2010; 26:871-8.

4. Mandelia A, Agarwala S, Sharma N, Solanki S, Panda S, Leftsided gastroschisis: A rare congenital anomaly. J Clin Diagn Res. 2013;7:2300-2.

5. Feldkamp ML, Carey JC, Sadler TW. Development of gastroschisis: review of hypothesis, a novel hypothesis, and implications for research. Am J Med Genet A. 2007; 143A:639-52. 